\title{
Digitale Vorlesungsformate. Ergebnisse einer semesterbegleitenden Untersuchung der Task Force Digitale Lehre des Instituts für Politische Wissenschaft und Soziologie der Universität Bonn
}

\author{
Manuel Becker · Jacqueline Fank • Theresa Gerlach • Felix Leßke • \\ Enrico Liedtke · Jakob Horneber • Tim Huyeng • Shushanik Minasyan • \\ Hendrik W. Ohnesorge $\cdot$ Yvonne Scheit $\cdot$ David Schmitz
}

Angenommen: 29. Juli 2021 / Online publiziert: 24. August 2021

(C) Der/die Autor(en) 2021

\section{Einleitung}

$\mathrm{Zu}$ Beginn der Corona-Pandemie traf die Herausforderung, Lehre im Sommersemester 2020 flächendeckend digital anbieten zu müssen, die deutschen Hochschulen in weiten Teilen unvorbereitet. Der äußere Druck führte vielerorts jedoch zu einem rasanten Lernprozess bei Lehrenden wie Lernenden, sodass die Hochschulen deutlich besser gerüstet in das Wintersemester 2020/21 gehen konnten. Hatte man vielerorts noch lange Zeit gehofft, wieder, wenn nicht flächendeckend, so doch zumindest zu großen Teilen, Präsenzlehre anbieten zu können, wurden diese Erwartungen schnell enttäuscht. Die Inzidenzwerte stiegen bereits seit Anfang August wieder, sodass schon im September an durchgehende Präsenzlehre nicht zu denken war - zumal in Nordrhein-Westfalen der Beginn des Wintersemesters auf Anfang November verschoben worden war. Zwar gab es zu Beginn des Semesters für einige wenige Wochen in ausgewählten kleinen Kursen Präsenzlehre, das Gros der Lehrveranstaltungen fand jedoch digital statt. Die wenigen Präsenzveranstaltungen wurden schließlich Anfang Dezember, bedingt durch das fortschreitende Infektionsgeschehen, nahezu vollumfänglich in den digitalen Raum verlagert. Und dennoch: Die Erfahrungen des ersten Digitalsemesters (vgl. Becker et al. 2020) konnten nun mit in die Vorbereitung der Lehre einfließen. Was im vergangenen Sommersemester 2020 noch vielerorts überstürzt und aus der Not heraus geboren worden war, konnte nun mit einem umfassenderen Erfahrungsschatz und mit deutlich größerem zeitlichen Vorlauf systematisch angegangen werden. Nichtsdestoweniger soll nicht

Manuel Becker $(\bowtie) \cdot$ Jacqueline Fank · Theresa Gerlach · Felix Leßke · Enrico Liedtke .

Jakob Horneber · Tim Huyeng · Shushanik Minasyan · Hendrik W. Ohnesorge · Yvonne Scheit ·

David Schmitz

Institut für Politische Wissenschaft und Soziologie, Rheinische Friedrich-Wilhelms-Universität

Bonn, Lennéstr. 25, 53113 Bonn, Deutschland

E-Mail: manuel.becker@uni-bonn.de 
unerwähnt bleiben, dass die lange unklare Lage vielfach zu Unsicherheiten in der Vorbereitung der Lehre führte.

Bei einem Veranstaltungstyp allerdings stand bereits seit dem Sommer 2020 fest, dass er im digitalen Raum würde stattfinden müssen: die Vorlesungen. Aufgrund der hohen Teilnehmer*innenzahlen in diesem Format war klar, dass das Vorlesungsangebot vollständig digital würde ausgerichtet sein müssen. Da die Studienstruktur am Institut für Politische Wissenschaft und Soziologie der Universität Bonn die großen Einführungsvorlesungen turnusgemäß für das Wintersemester vorsieht, hat die institutseigene Task Force Digitale Lehre diesen Veranstaltungstyp als Schwerpunkt der semesterbegleitenden Untersuchung gewählt. Neben regelmäßigen Reflexionsrunden wurde hierzu eine Umfrage unter den Studierenden gestartet, um die verschiedenen Varianten und Elemente zu evaluieren und für die Zukunft weiterzuentwickeln. Im Folgenden soll zunächst ein Überblick über die verschiedenen verwendeten Formate gegeben werden (Abschn. 2). Im Anschluss daran werden die Ergebnisse der Umfrage sowohl quantitativ (Abschn. 3) als auch qualitativ (Abschn. 4) ausgewertet, bevor die wesentlichen Erkenntnisse im Rahmen eines Fazits (Abschn. 5) zusammengefasst werden. In diesem Zusammenhang soll auch ein Ausblick auf die Zukunft digitaler Lehrinhalte nach der Pandemie gewagt werden.

\section{Digitale Vorlesungsformate im Überblick}

Auf einer grundsätzlichen Ebene lassen sich synchrone von asynchronen Formaten unterscheiden. Konkret bedeutet dies, dass die Vorlesungen entweder live via Videokonferenz abgehalten oder vorab aufgezeichnet und den Studierenden dann (schrittweise oder komplett) über digitale Lernplattformen zur Verfügung gestellt wurden. Auch Kombinationsformate aus beiden Typen sind vorstellbar. Im Sommersemester 2020 und Wintersemester 2020/21 fanden am Bonner Institut für Politische Wissenschaft und Soziologie insgesamt acht Vorlesungen mit begleitenden Übungen statt:

Die Vorlesung Einführung in die Politische Wissenschaft und Soziologie wurde komplett synchron abgehalten. Die beiden Dozenten waren bestrebt, die Vorlesung aus dem analogen Raum so authentisch, nah und gut wie möglich in den digitalen zu übertragen. Dazu wurde die Webinar-Funktion des Videokonferenzanbieters Zoom genutzt. Diese erlaubt neben der handlichen Verwaltung von sehr großen Teilnehmer*innenkreisen, die Studierenden zwischendurch für Rückfragen zu Wort kommen zu lassen. So konnten zumindest in bescheidenem Rahmen interaktive Elemente in einem ansonsten klassisch frontalen Veranstaltungsformat eingesetzt werden. Zudem wurde zur Vorbereitung der Sitzungen u. a. die Blogfunktion auf der digitalen Lernplattform der Universität Bonn (eCampus) genutzt, um inhaltliche Fragen bereits vorab von den Studierenden diskutieren zu lassen. Dieses Element wurde erstaunlich häufig von den Studierenden angenommen. Die begleitenden Übungen fanden größtenteils digital statt und waren nur lose mit der Vorlesung verbunden, da deren primäres Lernziel in der Propädeutik und in der Aneignung wissenschaftlicher Arbeitstechniken bestand, für die dieses Mal allerdings auch entsprechende Begleitvideos aufgezeichnet wurden. 
Im Falle der Vorlesung Demokratische und nichtdemokratische Herrschaftsformen, die zum Basismodul Politische Systeme gehört, waren die Übungen in die Vorlesung integriert. Die Vorlesung war in zehn Lehreinheiten unterteilt, zu denen jeweils ein 30- bis 60-minütiges Lehrvideo auf eCampus angeboten wurde. Ergänzt wurde dies um die Präsentationen sowie weiterführende Literatur auf eCampus. Die Lehreinheiten waren in drei thematisch zusammenhängende Blöcke unterteilt, nach denen jeweils eine Zoom-Konferenz als synchrone, interaktive Übung angeboten wurde, zu denen im Vorfeld Fragen per E-Mail oder über eCampus eingereicht werden konnten. Die Inhalte dieser Sitzungen wurden den Studierenden im Anschluss als Protokoll über eCampus zur Verfügung gestellt. Abgerundet wurde die Vorlesung durch einen Gastvortrag sowie eine synchrone Abschlusssitzung ebenfalls via Zoom.

In den Vorlesungen Einführung in die Statistik im Sommersemester 2020 und Methoden der empirischen Sozialforschung im Wintersemester 2020/21 wurden sowohl synchrone als auch asynchrone Elemente verwendet. Für jede Vorlesung wurden ein bis zwei Texte zur Vorbereitung bereitgestellt, deren Themen in der Vorlesung mit weiterführenden Beispielen behandelt wurden. Fragen zum Thema konnten vorab per Mail, aber auch im Rahmen der Vorlesung gestellt werden. Dies geschah entweder über Wortbeiträge oder über die Chat-Funktion von Zoom. Im Chat konnten sich die Studierenden die Fragen auch gegenseitig beantworten und austauschen. Wurde eine Frage nicht in diesem Austausch unter den Studierenden beantwortet, ging der Dozent auf die Inhalte ein. Die Vorlesung Einführung in die Statistik wurde zudem von Übungen und Tutorien begleitet. Für die Übungen wurden VideoPodcasts erstellt, in denen besonders praktische Inhalte der sozialwissenschaftlichen Statistik vermittelt wurden. Die Themen der Video-Podcasts wurden in synchronen digitalen Übungssitzungen besprochen und anhand praktischer Beispiele im Plenum diskutiert. Die Tutorien stellten ein freiwilliges Zusatzangebot dar. Hier wurden Übungsaufgaben gerechnet und besprochen.

Die Vorlesung Theorien der internationalen Beziehungen wurde in synchronem Format während der wöchentlich festgelegten Veranstaltungszeit über die VideoPlattform Zoom in Form eines Webinars abgehalten. Die Präsentationsfolien und später zusammenfassende Übersichten der jeweiligen Sitzungen - zu Fragen von Theorien \& Theoriebildung, Epistemologie \& Ontologie sowie, schwerpunktmäßig, ausgewählten Theorien der internationalen Beziehungen - wurden im Nachgang über die Plattform eCampus zur Verfügung gestellt. Um das Vorlesungsformat zu wahren und gleichzeitig einen interaktiven Austausch mit den Studierenden zu ermöglichen, fanden veranstaltungsbegleitend sowohl die Chat-Funktion als auch die Hand-Heben-Funktion von Zoom Verwendung, was stets ausgiebig genutzt wurde. Die zugehörigen Übungen im Basismodul Internationale Beziehungen waren eng mit der Vorlesung verzahnt: Zum einen wurden Inhalte der Vorlesung in wöchentlich digital stattfindenden Sitzungen basierend auf der vorherigen Lektüre ausgewählter Texte vertieft. Zum anderen lag ein besonderer Schwerpunkt auf der Transferleistung und eigenständigen Anwendung der erlernten Inhalte. Dies wurde u. a. durch Vorträge, regelmäßige Gruppenarbeiten (etwa durch Break-Out-Rooms in Zoom) und die Nutzung der Feedback-Funktion auf eCampus in abwechslungsreicher Art und 
Weise erreicht. Im Rahmen der Übungen wurden zudem synchrone und asynchrone Elemente kombiniert.

Die Vorlesung Einführung in die Politische Theorie und Ideengeschichte wurde vorab aufgezeichnet und den Studierenden jeweils am Vorabend der Sitzungstermine über die Lernplattform eCampus zur Verfügung gestellt, auf der sie über das gesamte Semester abrufbar blieb. Jede wöchentlich eingestellte Vorlesung teilte sich in drei bis vier thematisch zusammenhängende Videos von jeweils 15-20 min. Über die Orientierung an paarweise gruppierten Leitbegriffen und zugehörigen Problemfeldern nahm die Einführungsvorlesung einen umfassenden Blick auf die Geschichte des politischen Denkens ein. Begleitet wurde die Vorlesung durch wöchentliche Übungen, in denen ausgewählte Inhalte der Vorlesung entlang der gemeinsamen Textlektüre zentraler Autor*innen vertieft wurden. Vorlesung und Übung stellten daher einander ergänzende Formate dar.

Die Vorlesung Soziologische Theorie war so gestaltet, dass jede Woche (das ganze Semester über inkl. Einführungssitzung) vorab aufgenommene Lehrvideos über eCampus bereitgestellt wurden. Nur die Abschlusssitzung zur Klausurvorbereitung fand synchron über Zoom statt. Die Videos hatten eine Länge zwischen 45 und 135 min pro Sitzung. Zusätzlich dazu gab es zu jeder Sitzung einen Sekundärtext und ein bis zwei Primärtexte, die vorher oder nachher gelesen werden konnten. Die Lehre war insofern vor allem asynchron organisiert, wobei jede Woche während der offiziellen Vorlesungszeit eine parallel stattfindende Vorlesungssprechstunde angeboten wurde. Diese fand über Zoom statt und war als Gespräch geplant, bei dem die Studierenden ihre Rückfragen anbringen konnten. Diese vorlesungsbegleitenden Sprechstunden waren nicht obligatorisch und wurden nicht von allen Studierenden besucht.

Das Basismodul Deutsche und Europäische Politik bestand aus zwei Veranstaltungseinheiten. Die Vorlesung Europäische Integration: Interessen - Prozesse - Politiken wurde durch eine wöchentliche Übung begleitet, in der die Inhalte der Vorlesung aus einer spezifisch europäischen Perspektive vertieft und ergänzt wurden. Der Schwerpunkt des Moduls lag grundsätzlich auf synchronen Lehrmethoden und beide

Tab. 1 Übersicht der Lehrformate

\begin{tabular}{|c|c|c|}
\hline Modul & Vorlesung & Übung \\
\hline $\begin{array}{l}\text { Einführung in die Politische Wissen- } \\
\text { schaft und Soziologie }\end{array}$ & $\begin{array}{l}\text { Synchron (Zoom-Webinar) } \\
\text { begleitend: Blog }\end{array}$ & $\begin{array}{l}\text { Überwiegend } \\
\text { synchron }\end{array}$ \\
\hline Politische Systeme & $\begin{array}{l}\text { Asynchron (Lehrvi- } \\
\text { deos + Begleitmaterial) }\end{array}$ & $\begin{array}{l}\text { Synchron/ } \\
\text { integriert }\end{array}$ \\
\hline Methoden & Synchron/interaktiv (Zoom + Chat) & $\begin{array}{l}\text { Synchron/ } \\
\text { asynchron }\end{array}$ \\
\hline Internationale Beziehungen & $\begin{array}{l}\text { Synchron/interaktiv (Zoom-Webi- } \\
\text { nar+ Chat) + Begleitmaterial }\end{array}$ & $\begin{array}{l}\text { Synchron/ } \\
\text { asynchron }\end{array}$ \\
\hline Politische Theorie \& Ideengeschichte & Asynchron (Lehrvideos) & $\begin{array}{l}\text { Synchron } \\
+ \text { Begleitmaterial }\end{array}$ \\
\hline Allgemeine Soziologie & $\begin{array}{l}\text { Asynchron (Lehrvideos) + synchrone } \\
\text { Sprechstunde }\end{array}$ & $\begin{array}{l}\text { Synchron/ } \\
\text { asynchron }\end{array}$ \\
\hline Deutsche und Europäische Politik & Synchron (Zoom) & Synchron \\
\hline
\end{tabular}


Veranstaltungen wurden über Zoom durchgeführt. Für beide Veranstaltungen wurden separate Primärtexte ausgewählt, die vor jeder Sitzung gelesen werden konnten. Die Folien wurden ebenfalls bei eCampus bereitgestellt. Während der Übung wurde die Gelegenheit genutzt, die inhaltlichen Fragen aus der Vorlesung näher zu besprechen. Ferner wurde eine Online-Exkursion mit dem Verbindungsbüro des Europäischen Parlaments in Deutschland in die Übung integriert. Während der vierstündigen Online-Tour konnten die Studierenden aktuelle politische Themen mit europäischen Abgeordneten diskutieren.

Bezüglich des hier fokussierten Lehrformats der Vorlesung (inklusive begleitender Übungen) lässt sich eine Überblickstabelle erstellen (siehe Tab. 1).

\section{Quantitative Auswertung der Umfrage}

Zum Ende des Wintersemesters 2020/21 wurde unter den Studierenden eine Umfrage mit dem Ziel durchgeführt, Erfahrungswerte hinsichtlich digitaler Lehrformate mit dem Schwerpunkt auf dem Veranstaltungstypus ,Vorlesung“ zu sammeln, um so vergangene Lehre zu evaluieren und zukünftige Angebote zu verbessern. Hierzu wurde das Online-Umfragetool Questback verwendet. Nach dem Ende der Vorlesungszeit wurden sowohl Studierende des Bachelorstudiengangs „Politik und Gesellschaft“ als auch Studierende des Masterstudiengangs „Soziologie“ am Institut für Politische Wissenschaft und Soziologie der Universität Bonn per E-Mail zur Teilnahme aufgefordert.

Da das Interesse auf dem Veranstaltungstypus „Vorlesung“ lag, richtete sich die Umfrage nur an Studierende, die im Sommersemester 2020 oder im Wintersemester 2020/21 an mindestens einer Vorlesung teilgenommen haben. Insgesamt nahmen an der Umfrage 188 Studierende teil, die Stichprobe aller Befragten, die im betreffenden Zeitraum mindestens eine Vorlesung am Institut für Politische Wissenschaft und

Tab. 2 Überblick Vorlesungsbesuche der Umfrageteilnehmer*innen

\begin{tabular}{ll}
\hline Vorlesung & $\begin{array}{l}\text { Teilneh- } \\
\text { mer*innen }\end{array}$ \\
\hline Methoden der empirischen Sozialforschung (B.A. Politik und Gesellschaft) (SoSe 2020) & 53 \\
Einführung in die Statistik (B.A. Politik und Gesellschaft) (WiSe 2020/21) & 28 \\
Einführung in die Politikwissenschaft und Soziologie (B.A. Politik und Gesellschaft) & 71 \\
(WiSe 2020/21) & 60 \\
$\begin{array}{l}\text { Demokratische und nichtdemokratische Herrschaftsformen (B.A. Politik und Gesell- } \\
\text { schaft) (WiSe 2020/21) }\end{array}$ & \\
Theorien der internationalen Beziehungen (B.A. Politik und Gesellschaft) (WiSe 2020/21) & 64 \\
$\begin{array}{l}\text { Einführung in die politische Theorie und Ideengeschichte (B.A. Politik und Gesellschaft) } \\
\text { (WiSe 2020/21) }\end{array}$ & 58 \\
$\begin{array}{l}\text { Europäische Integration: Interessen - Prozesse - Politiken (B.A. Politik und Gesellschaft) } \\
\text { (WiSe 2020/21) }\end{array}$ & 32 \\
Soziologische Theorie (B.A. Politik und Gesellschaft) (WiSe 2020/21) & 56 \\
Cluster- und Skalierungsverfahren (M.A. Soziologie) (SoSe 2020) & 10 \\
Fortgeschrittene Regressionsanalyse (M.A. Soziologie) (WiSe 2020/21) & 10 \\
\hline
\end{tabular}


Soziologie belegt haben, beträgt 154 gültige Interviews. Davon belegten 19 Personen mindestens eine Vorlesung im Masterstudiengang. Tab. 2 gibt eine Übersicht über die Verteilung auf die von den Befragten besuchten Vorlesungen.

Insgesamt haben die Studierenden im Durchschnitt etwa drei Vorlesungen besucht. Die folgenden Angaben beziehen sich somit nicht auf eine einzelne Vorlesung, sondern geben einen modulübergreifenden Gesamteindruck wieder.

Im Vergleich zur Lehre vor Ort bietet die digitale Lehre besonders bei überwiegend passiv konsumierten Veranstaltungsformaten die Möglichkeit, diese an verschiedenen Orten und unter unterschiedlichen Bedingungen wahrzunehmen. Um dem auch in der Konzeption von Vorlesungen Rechnung tragen zu können, ist es von besonderem Interesse, das entsprechende Nutzungsverhalten der Studierenden kennenzulernen. Die Umfrage zeigt indes überraschend klassische Studiergewohnheiten. Fast alle Befragten gaben an, die angebotenen Inhalte vorrangig Zuhause am Schreibtisch zu konsumieren $(97,4 \%)$. Da bei dieser Frage Mehrfachantworten möglich waren, zeigte sich aber, dass die Teilnahme an den Vorlesungen auch von anderen Orten aus stattfindet: Dies können unter anderem das Sofa, das Bett oder ein Sessel sein (24,8\%), der Küchen- oder Esstisch (13,7\%), der Garten (5,9\%). Einige nahmen auch von unterwegs oder aus dem Ausland teil $(3,9 \%)$. Öffentliche Arbeitsplätze wurden hingegen in der Corona-Zeit relativ selten genutzt (1,3\%). Der Großteil der Befragten gab zudem an, den Inhalten überwiegend volle Konzentration zu widmen und auf anderweitige zeitgleiche Aktivitäten zu verzichten. 15,1\% der Befragten gaben an, z. T. nebenher Haushaltsaktivitäten auszuführen, 5,3\%, dass sie die Angebote zusammen mit Freund*innen oder Kommiliton*innen konsumieren und $2,0 \%$ betätigten sich zeitgleich sportlich.

Bei asynchronen Lehrangeboten ergab sich für die Studierenden zudem eine zeitliche Flexibilität, die auch genutzt wurde. Während ein Großteil der Befragten zwar vorrangig den vorgeschlagenen Bearbeitungszeitplan nutzte $(37,6 \%)$, bearbeiteten immerhin 30,8\% die Inhalte später als vorgeschlagen. Mit 26,3\% gab hier zudem auch ein Viertel an, keinem festgelegten Bearbeitungsschema zu folgen (,Mal so, mal so"). Unabhängig davon orientierte sich eine deutliche Mehrheit an den vorgeplanten Vorlesungseinheiten. So gaben $65,4 \%$ der Studierenden an, die jeweiligen Inhalte am Stück bearbeitet/angesehen/angehört zu haben. Etwa ein Drittel der Befragten nutzte zumindest teilweise die Möglichkeit, asynchrone Inhalte in mehreren Etappen zu bearbeiten. Auch die nur in der asynchronen Lehre bestehende Option, die zur Verfügung gestellten Lerninhalte mehrfach nutzen zu können, wurde nur von einer Minderheit genutzt. Ein großer Teil der Befragten $(61,7 \%)$ hat davon keinen Gebrauch gemacht. Lediglich ein knappes Drittel hat auf die Inhalte zweimal $(29,3 \%)$, etwa $8,3 \%$ sogar dreimal oder öfter zugegriffen. Dabei wurden die asynchronen Inhalte in den höheren Fachsemestern tendenziell häufiger abgerufen.

Die im Sommersemester 2020 und Wintersemester 2020/21 am Institut für Politische Wissenschaft und Soziologie angebotenen Vorlesungen waren zum Teil deutlich unterschiedlich konzipiert. Insbesondere wurden synchrone und asynchrone Elemente in unterschiedlichen Graden genutzt. Gefragt nach dem Format der besuchten Vorlesungen gaben 37,9\% der Befragten an, dass dieses vollständig oder vorwiegend synchron gewesen sei. 42,5\% antworteten hier "teils teils“. Hingegen gaben nur $19,6 \%$ an, die Vorlesungen hätten vorrangig oder vollständig asynchron stattge- 
Tab. 3 Bisherige Veranstaltungsformate und zukünftige Präferenzen

\begin{tabular}{lllllll}
\hline & Präferenz & & & & \\
& & & & \\
Vollständig & Vorwiegend & Teils & Vorwiegend & Vollständig \\
synchron & synchron & teils & asynchron & asynchron \\
Bisherige & Vollständig & $36,8 \%$ & $31,6 \%$ & $5,3 \%$ & $15,8 \%$ & $10,5 \%$ \\
tungen & synchron & $(7)$ & $(6)$ & $(1)$ & $(3)$ & $(2)$ \\
& Vorwiegend & $25,6 \%$ & $41,0 \%$ & $17,9 \%$ & $10,3 \%$ & $5,1 \%$ \\
& synchron & $(10)$ & $(16)$ & $(7)$ & $(4)$ & $(2)$ \\
& Teils teils & $12,3 \%$ & $24,6 \%$ & $29,2 \%$ & $29,2 \%$ & $4,6 \%$ \\
& & $(8)$ & $(16)$ & $(19)$ & $(19)$ & $(3)$ \\
& Vorwiegend & $4,2 \%$ & $8,3 \%$ & $29,2 \%$ & $37,5 \%$ & $20,8 \%$ \\
& asynchron & $(1)$ & $(2)$ & $(7)$ & $(9)$ & $(5)$ \\
& Vollständig & $0,0 \%$ & $16,7 \%$ & $66,7 \%$ & $0,0 \%$ & $16,7 \%$ \\
& asynchron & $(0)$ & $(1)$ & $(4)$ & $(0)$ & $(1)$ \\
& & Chi-Qua- & 39,8 & & Cramérs V & 0,26 \\
& & drat & $(\mathrm{p}<0,01)$ & & & \\
\hline
\end{tabular}

funden. Vergleichbar breit fallen auch die Studierendenpräferenzen aus. Die Frage nach den jeweils präferierten Vorlesungsformaten zeigt dabei, dass der Wunsch nach überwiegend asynchronen Inhalten, mit 31,4\% deutlich höher als das tatsächliche Angebot war. Der Anteil der Studierenden, die sich vorwiegend oder vollständig synchrone Lehre wünschen liegt mit 43,8\% jedoch nochmals klar über diesem Wert.

Angesichts des sehr diversen Bildes der Erfahrungen und Erwartungen hinsichtlich synchroner oder asynchroner Inhalte stellt sich die Frage, ob sich aus den jeweils bekannten Lehrformaten Folgen für künftige Präferenzen ergeben. Aus diesem Grund haben wir die Variablen des Lehrformats der bisher besuchten Vorlesungen dem präferierten Lehrformat in einer Kreuztabelle gegenübergestellt (Tab. 3).

Wie in der Kreuztabelle zu erkennen ist, werden überwiegend die Formate als Präferenz angegeben, mit denen die Studierenden auch vorrangig zu tun hatten. So gaben $68,4 \%$ der Befragten, die vollständig asynchron unterrichtet wurden, an, dass sie auch zukünftig eine vollständige oder vorwiegende synchrone Lehre bevorzugen würden. Auch bei den Studierenden, die vorwiegend synchron unterrichtet wurden, besteht zu 66,6\% eine eindeutige Präferenz für synchrone Lehrformate. Auf der anderen Seite des Spektrums zeigt sich hingegen, dass die Studierenden, die vorwiegend asynchron unterrichtet wurden, ebenfalls mit einer eindeutigen Mehrheit von 58,3\% asynchrone Lehrformate bevorzugen. Im Gegensatz zu den Angaben zur synchronen Lehre ist hier jedoch die Mittelkategorie ,teils teils“ relativ gesehen etwas stärker besetzt. Auch von den sechs Personen, die angaben, vollständig asynchron unterrichtet worden zu sein, findet sich eine stärkere Tendenz hinsichtlich der Mittelkategorie (66,7\%). Insgesamt scheinen die erlebten Lehrformate generell als positiv wahrgenommen worden zu sein. Der Zusammenhang zwischen beiden Variablen ist auf dem 1\%-Niveau signifikant. Mit einem Cramérs V von 0,26 ist der Effekt für sozialwissenschaftliche Verhältnisse zudem relativ stark. Es kann daraus geschlossen werden, dass es für die Studierenden möglicherweise gar nicht von zentraler Bedeutung ist, ob synchrone oder asynchrone Formate angeboten werden, solange diese gut gestaltet sind. 
Tab. 4 Fachsemester und Formatspräferenzen

\begin{tabular}{llllll}
\hline $\begin{array}{l}\text { Fach- } \\
\text { semester }\end{array}$ & $\begin{array}{l}\text { Präferenz } \\
\text { Vollständig } \\
\text { synchron }\end{array}$ & $\begin{array}{l}\text { Vorwiegend } \\
\text { synchron }\end{array}$ & $\begin{array}{l}\text { Teils } \\
\text { teils }\end{array}$ & $\begin{array}{l}\text { Vorwiegend } \\
\text { asynchron }\end{array}$ & $\begin{array}{l}\text { Vollständig } \\
\text { asynchron }\end{array}$ \\
\hline $1 . / 2$. & $23,5 \%$ & $34,1 \%$ & $22,4 \%$ & $16,5 \%$ & $3,5 \%$ \\
& $(20)$ & $(29)$ & $(19)$ & $(14)$ & $(3)$ \\
3. & $7,7 \%$ & $23,1 \%$ & $32,7 \%$ & $26,9 \%$ & $9,6 \%$ \\
& $(4)$ & $(12)$ & $(17)$ & $(14)$ & $(5)$ \\
$4 . /$ & $12,5 \%$ & $0,0 \%$ & $12,5 \%$ & $43,8 \%$ & $31,3 \%$ \\
höheres & $(2)$ & $(0)$ & $(2)$ & $(7)$ & $(5)$ \\
& Chi-Quadrat & 30,9 & & Cramérs V & 0,32 \\
& & $(\mathrm{p}<0,001)$ & & & \\
\hline
\end{tabular}

Welche Vorlesungen belegt wurden und mithin, welche Erfahrungen gemacht werden konnten, hängt indes nicht zuletzt vom Studienfortschritt ab. Insofern liegt es nahe, einen Zusammenhang zwischen der Lehrpräferenz und dem jeweiligen Fachsemester der Studierenden zu vermuten (Tab. 4).

Die Kreuztabellierung beider Variablen zeigt, dass vor allem im ersten und zweiten Semester synchrone Formate präferiert werden (57,6\%). In höheren Semestern hingegen werden zunehmend asynchrone Formate bevorzugt. Der Zusammenhang der beiden Variablen ist auf dem 0,1\%-Niveau signifikant, mit einem Cramérs V von 0,32 fällt der Effekt auch statistisch relativ stark aus. Neben einer signifikanten Präferenz für die Lehrformate, die man selbst bereits kennengelernt hat, ist für diesen Zusammenhang auch eine weitere Erklärung denkbar: Asynchrone Formate bieten mehr Flexibilität, erfordern aber auch eine bessere Selbstorganisation. Sie sind damit vermutlich vor allem für Studierende in höheren Semestern attraktiv, die mit dem Studium im Allgemeinen und der Selbstorganisation bereits stärker vertraut sind als Erst- oder Zweitsemester. Eine gesonderte Betrachtung der Masterstudierenden ergab, dass auch hier die Präferenz der Erst- und Zweitsemester in Richtung synchrone Formate, bei höheren Fachsemestern in Richtung asynchroner Formate geht.

Als weiterer erklärender Faktor drängt sich die Tatsache auf, dass Erst- und Zweitsemester zum Zeitpunkt der Befragung noch gar keine Gelegenheit hatten, nichtdigitale Unterrichtsformate am Institut für Politische Wissenschaft und Soziologie der Universität Bonn kennenzulernen. Mehr noch, hatten sie bisher nur sehr eingeschränkt die Möglichkeit, persönlichen Kontakt zu ihren Kommiliton*innen und Dozent*innen aufzunehmen. Insofern könnten sich synchrone Formate in dieser Gruppe nicht zuletzt auch deshalb hoher Beliebtheit erfreuen, weil sie die Möglichkeit bieten, einen Eindruck der Dozent*innen und - vielleicht noch wichtiger - der Kommiliton*innen zu erlangen. Sofern die pandemische Lage auch in den kommenden Semestern keine Präsenzlehre zulassen sollte, kann demzufolge vermutet werden, dass die verstärkte Präferenz für synchrone Lehrformate in diesen Jahrgängen, die bisher nur digitale Lehre erlebt haben, auch in höheren Fachsemestern bestehen bleibt. 


\section{Qualitative Auswertung der Umfrage}

Mit dem in die Umfrage integrierten offenen Frageblock sollte ein breites Erfahrungsbild der Studierenden hinsichtlich der digitalen Vorlesungsformate eingeholt werden. Dazu wurde zunächst nach den Vor- und Nachteilen digitaler Vorlesungsformate gefragt. Unter den Befragten gaben mehr Studierende Vorteile $(n=124)$ als Nachteile $(n=102)$ an. Grundsätzlich zeigt die Auswertung der Antworten auf die Frage nach den Vorteilen digitaler Vorlesungsformate, dass diese insbesondere bei den asynchronen Formaten gesehen werden. Als am häufigsten genannten Vorteil der aufgezeichneten Vorlesungen gaben die Studierenden die Möglichkeit der Wiederholung und des Pausierens von Inhalten innerhalb eines Videos an (75 Nennungen). Das mehrmalige Anhören der Vorlesungen helfe insbesondere bei der Lösung von Verständnisproblemen, aber auch bei der intensiven Nachbereitung einzelner Sitzungen. Auch zur Prüfungsvorbereitung sei das wiederholte Anhören zu einem späteren Zeitpunkt vorteilhaft, wenngleich die quantitative Auswertung gezeigt hat, dass diese Möglichkeit nur von einer Minderheit genutzt wurde. In diesem Kontext wurde die ständige Verfügbarkeit der Lehrinhalte seitens der Studierenden als äußerst positiv empfunden. Ferner ermögliche das Anhalten der Vorlesungsvideos das Führen ordentlicher und vollständiger Mitschriften sowie das Einlegen einer Pause, wenn die Konzentration abnimmt. Die selbstständige Bestimmung des eigenen Lerntempos sowie eine flexible Zeiteinteilung bei der Bearbeitung von Lehrinhalten wurden außerdem häufig als Vorteile des asynchronen Formates genannt (19 Nennungen).

Die Stichworte „Flexibilität“ und „Selbstorganisation“ trafen nach den Befragten jedoch nicht nur auf das asynchrone, sondern auf das digitale Vorlesungsformat insgesamt zu. So empfanden die Studierenden die zeitliche und räumliche Ungebundenheit als einen Zugewinn an Unabhängigkeit, Flexibilität und Freiheit in der Gestaltung des eigenen Lernalltags (44 Nennungen). Zusätzlich ließe sich durch das Arbeiten von zu Hause ein angenehmes Lernumfeld schaffen, indem ein „konzentrierteres [A]rbeiten viel leichter möglich [ist] als in einem Raum mit sehr vielen anderen Studenten“ (Fbn. 26) oder aber ,die Vorlesung mit Freunden im Wohnzimmer [gehört werden kann]“ (Fbn. 207).

Als größten Vorteil identifizierten die Studierenden jedoch Zeitersparnisse, die sich durch das digitale Format der Vorlesungen ergaben. Mehr als die Hälfte der Studierenden (66 Nennungen) gab an, durch das Wegfallen der Pendelfahrten zwischen Universität und Zuhause sowie der Wege zwischen Veranstaltungsorten Zeit zu gewinnen, die anderweitig gut genutzt werden könne - beispielsweise zur Vorund Nachbereitungszeit (Fbn. 92, 118, 195, 227), aber auch ,um länger schlafen zu können“ (Fbn. 153) oder „,wenns [sic!] regnet nicht zur Uni fahren zu müssen“ (Fbn. 306). Damit zusammenhängend berichteten die Studierenden, dass sich aufeinanderfolgende Veranstaltungen besser koordinieren ließen und die halbstündigen Pausen zwischen den Veranstaltungen zur tatsächlichen Erholung genutzt werden konnten, da stressige Raumwechsel entfielen. Zudem gaben elf Studierende an, durch die digitalen Vorlesungsformate Studium und Nebenjob und/oder Familie und Haushaltspflichten besser vereinbaren zu können. Ferner wurden als Vorteile des Digitalformates die Ermöglichung, auch bei Krankheit teilnehmen zu können (5 Nennungen) sowie die Diversität an Lehr- und Lernformaten (6 Nennungen) durch den 
Einsatz von „Lernvideos“, „Quizzen“, „Umfragen“ und „Break-out-sessions“ genannt (Fbn. 30, 43, 108). Bemerkenswert ist, dass es in mehreren Antworten auf die Frage nach den Vorteilen der digitalen Vorlesungsformate als besonders positiv herausgestellt wurde, wenn die Dozierenden Raum für das Stellen von Nachfragen gelassen haben (Fbn. 43, 179, 212). Dies verweist bereits auf den Wunsch seitens der Studierenden, asynchrone mit synchronen Formaten zu kombinieren.

Auf die offene Frage „Wo sehen Sie Nachteile oder Verbesserungsmöglichkeiten der digitalen Vorlesungsformate“ nannten 77,5\% der Studierenden ${ }^{1}$ fehlende Kontakte (allgemein sowie zu Kommiliton*innen und Dozent*innen). An zweiter Stelle, immerhin von jedem*r dritten Studierenden genannt, liegen Probleme der eigenen Lernsituation (Konzentrationsschwierigkeiten, Ablenkung, fehlende Motivation), direkt gefolgt von Kritik am Ablauf von live abgehaltenen Vorlesungen, in denen vor allem ein geringes Maß an Beteiligung und Interaktion sowie wenig Raum für Diskussionen kritisiert wurden. Auch wurde von 17,6\% der Studierenden angegeben, dass Nachteile der zeitlichen Organisation für sie entstanden - dass beispielsweise die Bearbeitung von Materialien lange dauerte oder das Tempo in Vorlesungen zu hoch war. Technische Probleme bei der eigenen Ausstattung, Monotonie am Schreib-

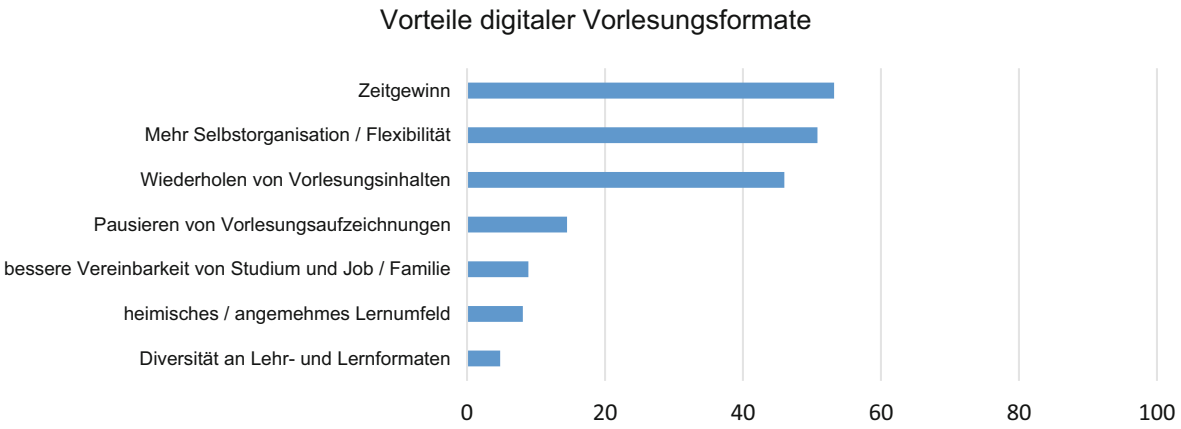

Nachteile digitaler Vorlesungsformate

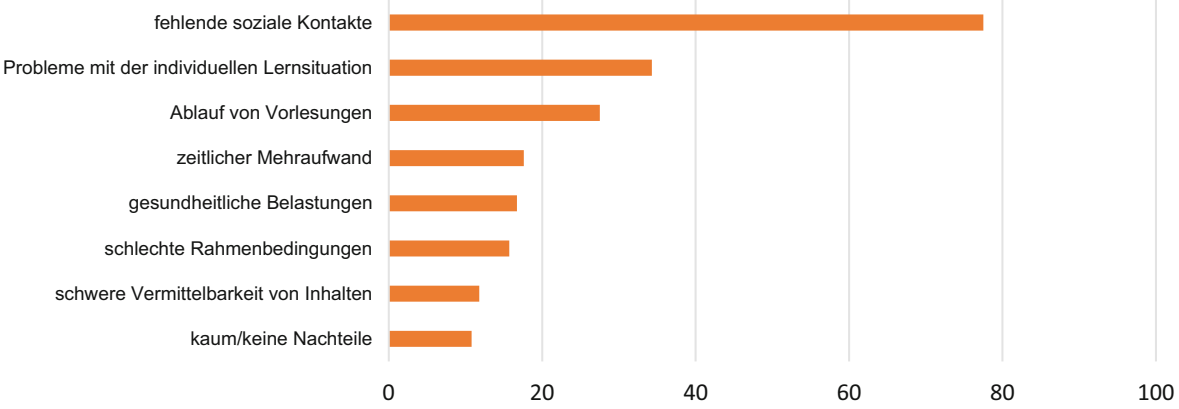

Abb. 1 Vorteile und Nachteile der digitalen Vorlesungsformate

\footnotetext{
1 Die Prozentzahl bezieht sich auf die Studierenden, die eine Angabe bei Nachteilen machten, also 77,5\% der 102 Studierenden.
} 
tisch, schlechte Wohn- und Lernbedingungen wurden unter ,Rahmenbedingungen “ subsumiert und von fast $16 \%$ der Studierenden als Nachteil angegeben. Dass Inhalte schwerer erlernt, weniger vertieft und allgemein weniger Lernstoff mitgenommen werden konnten, wurde von fast $12 \%$ der Studierenden als Nachteil empfunden. Jede*r zehnte Studierende gab an, keine oder kaum Nachteile empfunden zu haben (entweder explizit oder bei der offenen Frage wurden nur Vorteile genannt). Die im Rahmen der Umfrage erhobenen Vor- und Nachteile digitaler Vorlesungsformate lassen sich entsprechend in Abb. 1 zusammenfassen.

Neben der Codierung anhand der Überkategorien (jeweils ein Code pro Person und Überkategorie) wurde im Folgenden eine kleinteiligere Codierung der einzelnen genannten Aspekte vorgenommen. Es wurden dabei jeweils alle von einem*r jeweiligen befragten Studierenden angegebenen Aspekte codiert, auch wenn mehrere Aspekte innerhalb einer Überkategorie genannt wurden (z. B. bei Gesundheit sowohl psychische als auch physische Probleme). Durchschnittlich wurden etwas mehr als drei Aspekte, also drei Nachteile (326 genannte Aspekte bei 102 Antworten) pro Person genannt. Manche Studierende nannten nur ein oder zwei Stichworte, andere schrieben längere Texte. Die einzelnen als nachteilig empfundenen Aspekte sind in Abb. 2 dargestellt.

Hinsichtlich der Aussagen der Umfrageergebnisse verändert diese kleinteiligere Codierung nicht viel. Weiterhin wurden mit Abstand am häufigsten Aspekte des sozialen Austauschs und sozialer Kontakte sowie der Kommunikation genannt. Hierunter fallen drei große Bereiche: das Vermissen des Kontaktes, der Interaktion und des Austauschs mit Kommiliton*innen (74 Nennungen); das Fehlen des persönlichen Bezuges und Kontaktes mit den Dozent*innen (34 Nennungen); sowie das generelle Fehlen menschlicher Kontakte (15 Nennungen). Festgehalten werden muss, dass neben dem Bedauern, dass kein persönlicher Bezug zu den Dozent*innen

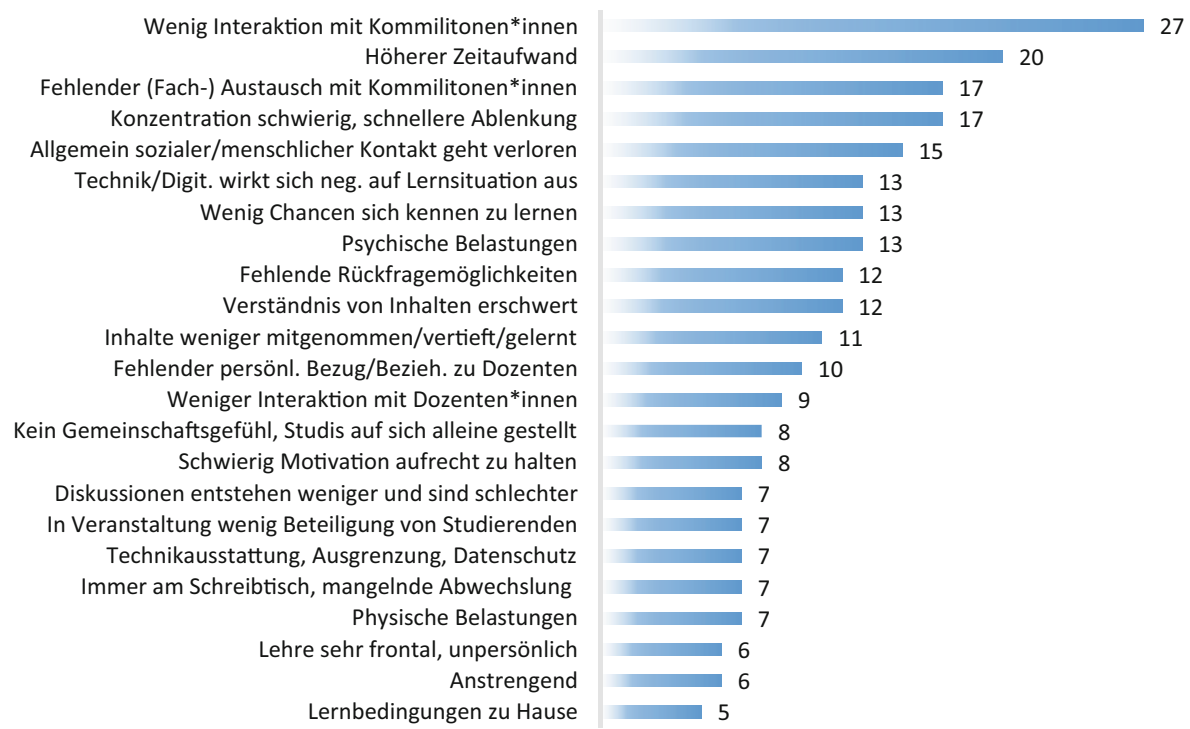

Abb. 2 Nachteile - Einzelne Aspekte 
hergestellt werden könne, immer wieder auch deutlich kritisiert wurde, wenn Fragen im Rahmen der Vorlesung nicht zugelassen wurden oder generell Unterstützung und Rückfragemöglichkeiten von Seiten der Dozent*innen fehlten. Zusatzangebote zu asynchronen Vorlesungen wurden zwar geschätzt, aber auch von einzelnen Studierenden als Mehraufwand wahrgenommen. Bei den 74 Nennungen in Bezug auf die fehlende soziale Komponente zwischen Studierenden wurden mit am häufigsten die fehlende Interaktion und Kommunikation mit Studierenden genannt (27 Nennungen), an zweiter Stelle vermissten viele Studierende den fachlichen oder persönlichen Austausch mit Kommiliton*innen (17 Nennungen), an dritter Stelle bedauerten viele Studierende, dass ihnen die Chance fehlte, neue Kontakte zu knüpfen oder Freundschaften aufzubauen (13 Nennungen).

Als zweithäufigster Aspekt in der Umfrage hinsichtlich der Nachteile des digitalen Vorlesungsformates wurde die individuelle Lernsituation genannt. Studierende hatten damit zu kämpfen, ihre Motivation aufrecht zu erhalten (8 Nennungen). Viele klagten (in abfallender Häufigkeit) über Unkonzentriertheit, Ablenkungen, ein als anstrengend empfundenes Lernen, weniger Leistung, weniger aktive Teilnahme und Frustration. Die Folge war teilweise Prokrastination (vor allem bei asynchronen Formaten) und ein geringeres Vertiefen und Lernen von Inhalten der Lehre bzw. des Studiums. Auch verspürten einige Studierende einen erhöhten Druck zum Selbstmanagement bzw. zur Selbstorganisation.

Bei der Bewertung des Ablaufs von (synchronen) Vorlesungen wurde am häufigsten die geringere oder sogar ganz fehlende Beteiligung und Interaktion von Seiten der Studierenden genannt; gleichzeitig wurde auch der mangelnde Raum für Diskussionen, viel Frontalunterricht und die damit stark monodirektionale Ausrichtung des Dozierens kritisiert. Auch der technische Aspekt spielte dabei eine Rolle. So wurden als Verbesserungsvorschläge am häufigsten technische Komponenten und die Gestaltungen von Sitzungen genannt, nämlich vor allem der Einbezug von Studierenden, das Einbauen von partizipativen Elementen in die Lehre und ein kreativerer und abwechslungsreicherer Umgang mit den digitalen Möglichkeiten. Vorgeschlagen wurde die Aufnahme von partizipativen Elementen wie u.a. Breakout-Rooms, Tests/Umfragen, Videos, und es wurden kleinere Pausen zur Auflockerung vorgeschlagen.

So scheint ein weiterer wichtiger Punkt die Vermittlung der Inhalte und das Arbeitspensum der Studierenden zu sein. Es wurde immer wieder deutlich kritisiert, dass es zu einem erheblichen zeitlichen Mehraufwand gekommen sei (20 Nennungen) - einerseits durch ein fehlendes Verständnis (Inhalte vor allem in asynchronen Vorlesungen zu komplex dargestellt, abstrakte Sprache in Vorlesungsfolien, fehlende Erklärungen) und andererseits durch fehlende Möglichkeiten zur Rückfrage, wodurch Folien oder generell Vorlesungsinhalte von Studierenden in hohem Zeitaufwand aufgearbeitet werden mussten. Dies wurde noch durch die fehlende soziale Ebene verschärft, sodass einige Studierende kritisierten, sich alleine gelassen zu fühlen. Gleichzeitig wurde auch moniert, dass manche synchron wie asynchron gehaltenen Vorlesungen sich nicht an das vorgesehene Zeitlimit von 90 min hielten. Studierenden fehlte die Orientierung, wichtige von unwichtigen Inhalten trennen zu können und durch die Komplexität, die hohen Erwartungen, den fehlenden sozialen Austausch und die zuvor beschriebenen Probleme der Lernsituation (Konzentrati- 
on, Motivation) gaben Studierende immer wieder an, aus den Vorlesungen weniger Inhalte mitzunehmen und vertiefen zu können sowie schlechter zu lernen.

Schlechte Rahmenbedingungen (fehlende technische Ausstattung, ungünstige Wohnverhältnisse) wurden nur wenige Male genannt, eigentlich überraschend selten im Vergleich zu allen anderen Nennungen. Nur drei Studierende von allen Befragten gaben an, Probleme mit Internet oder Technik zu haben. Als schwierig wurde eher die fehlende räumliche Trennung von Freizeit, Wohnen und Studieren empfunden. Durch das Studieren zu Hause am Schreibtisch wurde das Studium von einigen Studierenden als monoton und wenig abwechslungsreich beschrieben und oft zusammen mit Problemen der individuellen Lernsituation (wie oben beschrieben) genannt. Studierende empfanden, dass durch die technische Vermittlung Einschränkungen der Lehre und Lernsituationen entstehen (13 Nennungen).

Eng verbunden mit allen bisher genannten Aspekten gehen die psychischen und physischen Belastungen einher. Codiert wurden diese als solche, wenn die Belastung explizit genannt wurde, denn natürlich können alle vorher genannten Kritikpunkte auch zu Belastungen führen. Dabei scheint die psychische Ebene der Belastung (13 Nennungen) die der physischen (nur 7 Nennungen) zu überwiegen. Als psychische Belastungen gaben die Studierende Einsamkeit und Isolation, Stress, Depression, Selbstzweifel und fehlende Möglichkeiten zum Abschalten durch die fehlende Trennung von Studium und Freizeit an. Physisch empfanden sie den Bewegungsmangel und die lange Arbeit am Bildschirm als belastend.

Mit der Frage „Wenn Sie frei entscheiden könnten: Wie sähe für Sie ein optimales Lehrangebot aus?“" sollte den Studierenden ermöglicht werden, eine klare Rückmeldung ihrer erwünschten Lehrformate zu geben und somit die Reflexion über zukünftige Lehrplanung anzuregen. Nachdem bereits ein erster Eindruck über die Wünsche der Studierenden anhand der quantitativen Auswertung der geschlossenen Fragen entstehen konnte, zeigen die Antworten auf die offenen Fragen nun das beeindruckende Spektrum von individuellen Bedürfnislagen und Vorschlägen für die Zukunft. Allerdings kann die augenscheinliche Tendenz des ersten Teils des Fragebogens bestätigt werden: Die Studierenden neigen in ihrer Mehrheit eher zu einer in unterschiedlichen Modi eingelassenen Hybridlösung für die Lehre. Etwas seltener waren Forderungen nach eingleisiger Rückkehr zur Präsenzlehre ohne digitale Begleitung oder eben die völlige Umsetzung der Lehre durch asynchrone Digitalisate. Einen ersten Überblick kann eine rudimentäre quantitative Inhaltsanalyse der Antworten in Abb. 3 bieten.

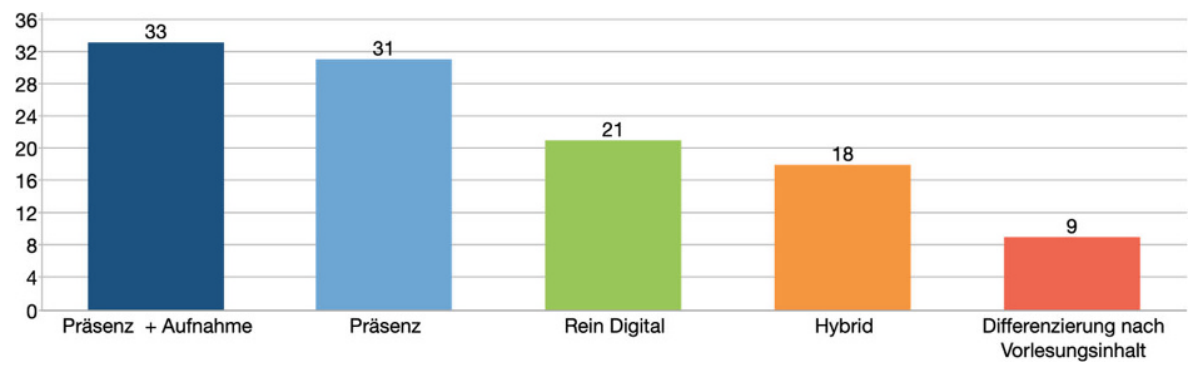

Abb. 3 Präferierte Vorlesungsformate für die Zukunft 
So einfach diese Grafik auf den ersten Blick erscheint, so vielfältig sind die darin subsumierten Antworten der Studierenden. Die Codierung war sehr komplex, da die Beantwortung einer so allgemein gestellten Frage teilweise zu narrativen Aussagen führte. Darüber hinaus wurde die Frage zum Teil unter dem Eindruck der quantitativen Befragung auf die Vorlesungsoptionen synchron/asynchron bezogen; häufig spielten aber auch allgemeinere Vorschläge zur Lehre im gesamten Studienfach eine Rolle. Aus insgesamt 64 Antworten, die die Form der Präsenzlehre für ihre universitäre Ausbildung ganz klar ins Zentrum stellen, lässt sich auf eine gewisse ,analoge Sehnsucht“ bei den Studierenden schließen. Der relativ größte Anteil der Befragten wünschten sich ein Vorlesungsformat, das sowohl in Präsenz im Vorlesungssaal stattfindet, als auch gleichzeitig für die spätere asynchrone Nachbearbeitung aufgenommen wird. In diesem Format wurden von vielen Befragten der bestmögliche Zusammenschluss von Vorteilen der Präsenz- wie Digitallehre gesehen, da sich menschliche Kontakte mit höherer Flexibilität vereinen und somit verschiedene Bedürfnisse befriedigen ließen. Weiterhin wurde in Bezug auf dieses Format die Möglichkeit des eigenständigen Nacharbeitens bei Fehlen durch Krankheit als Vorteil genannt (Fbn. 169). Gerade durch mangelnde Sozialisierungsmöglichkeiten mit anderen Kommiliton*innen während des Digitalsemesters könnte diese Selbstständigkeit für einige Studierende die einzige Möglichkeit darstellen, über die verpassten Inhalte informiert zu sein. In Zeiten weiter anhaltender pandemischer Gefahren seien so aber auch vulnerable Studierende zu integrieren, die trotz niedriger Inzidenzzahlen größere Menschenansammlung meiden müssten (vgl. Fbn. 160). Es wurden aber auch Bedenken in Bezug auf den Datenschutz geäußert (Fbn. 112, 129, 176).

Die unter dem Stichwort „Hybrid“ zusammengefassten Vorschläge einer optimalen Lehre enthielten häufig wenig konkretisierte Balancegewichtungen zwischen Präsenz- und Digitallehre. Zum Teil fiel es dabei schwer, nachträglich zu rekonstruieren, ob mit synchroner Lehre dann auch gleichzeitig Präsenzlehre eingeschlossen werden sollte beziehungsweise unter welcher Prämisse die Antworten gegeben wurden. Schließlich ließe sich die Antwort auf eine „Post-Corona-Zeit“, wie auch auf eine anhaltende pandemische Lage beziehen. Die Hybridlösungen beinhalteten aber zu einer überwiegenden Mehrheit (11 Antworten) den Wunsch nach einer digitalen Vorlesung und gleichzeitig analogen Übungen, die die Schwächen des Digitalen kompensieren sollten. Ähnlich argumentierten auch Studierende, die zwar für eine umfassende Digitalisierung der Vorlesungen eintraten, aber sich gleichzeitig Seminare in Präsenzlehre wünschten. ${ }^{2}$

Weiterhin wurde auch zwischen verschiedenen Vorlesungsinhalten differenziert. So war vor allem die Forderung nach der Fortdauer der Digitalisierung der Statistikvorlesung häufiger zu finden (s. Fbn. 30, 61, 218). Bei den komplexen Inhalten mit hoher Relevanz für die Modulabschlussklausur empfanden die Studierenden vor allem die Funktionen des Stoppens, Verlangsamens und Zurückspulens von digital verfügbaren Videos als hilfreich. Diese differenzierte Perspektive auf die Stimmigkeit von Form und Inhalte trifft dabei einen Kern aktueller pädagogischer Debatten,

\footnotetext{
${ }^{2}$ Diese Antworten wurden dennoch dem Code „Rein Digital“ zugerechnet, da sich die Umfrage zentral auf die Vorlesungen bezog.
} 
die den Fokus weniger auf die Anpassung der Lernformen auf personengebundene Lerntypen lenkt, sondern stärker auf die inhaltsbezogene Lerneinheiten (s. Wisniewski 2013; Looß 2019). Hier ließe sich über die Rückbindung an weitere pädagogische Forschung entsprechend eine rationale Zuweisung verschiedener Vorlesungen zu Lernformaten mit den Variablen Digital/Präsenz und Synchronität/Asynchronität vornehmen.

Ganz zentral erschien für die Studierenden ein aufgeräumter und großzügig gefüllter Kurs-Ordner auf der uniinternen Lernplattform eCampus. Der Wunsch nach einer erhöhten Pflege dieses asynchronen und konstant abrufbaren Online-Auftritts der Veranstaltungen (22 Antworten) war stets mit der Hoffnung verbunden, die Lernmaterialien mit höchster Effizienz abrufen zu können und somit Zeit und Energie auf wichtigere Aufgaben verwenden zu können. So nachvollziehbar der Wunsch ist, drückt sich hierin auch eine neue Erwartungshaltung aus, die die Universität immer stärker zu einem kundenorientierten Dienstleistungsunternehmen werden lassen. Stattdessen könnten durch die selbstständige Besorgung und Organisation der Inhalte Sekundärkompetenzen im Studium geschult werden. Diese Spannung zeigte sich auch in der Ambivalenz der Forderungen nach ,weniger Verschulung“ (Fbn. 92) auf der einen Seite und einem ,persönlichen Fahrplan“ (Fbn. 277) auf der anderen.

Ein praktischer Hinweis mit Blick auf die zukünftigen Semester, die eventuell tatsächlich digitale mit analogen Lehrangeboten kombinieren könnten, scheint noch angezeigt: „Wenn man sowohl Präsenz- als auch Online-Veranstaltungen wahrnehmen muss, wäre es aktuell schwierig, beiden ausreichend folgen zu können“ (Fbn. 302). Mehr vollständig ausgestattete Arbeitsplätze und Lernmöglichkeiten in der Universität könnten daher bei kurzfristigen Wechseln des Lehrformats notwendig werden.

Wie es sich bereits in der Beantwortung der geschlossenen Frageformate abzeichnete, lässt sich zusammenfassen: Die Studierenden wünschen sich mit klarer Mehrheit, dass die Universität ihre neu hinzugewonnen Kompetenzen weiterhin zum Wohle der Studierenden in der Lehre nutzt, diese aber gleichzeitig mit traditionellen Formaten kombiniert.

\section{Fazit und Ausblick}

Da im ersten Corona-Semester, dem Sommersemester 2020, am Institut für Politische Wissenschaft und Soziologie der Universität Bonn zwar bereits zahlreiche Seminare in digitalem Format durchgeführt wurden, aber nur einzelne Vorlesungen, gab es im erneut digitalen Wintersemester 2020/21 noch relativ wenige Erfahrungswerte bezüglich dieses Veranstaltungsformates. Gleichzeitig gehören Vorlesungen, gerade für Studienanfänger*innen, im Wintersemester traditionell zu den Pflichtveranstaltungen im politikwissenschaftlichen und soziologischen Studium in Bonn. Um die Kenntnisse darüber zu erweitern, wie die digital durchgeführten Vorlesungen von den Studierenden wahrgenommen wurden, und nicht zuletzt welche Lehren für zukünftige Veranstaltungsformate gezogen werden können, wurde von der Task Force Digitale Lehre eine Umfrage unter den Studierenden durchgeführt. Die zugrundeliegende Stichprobe der Studierenden, die im Sommersemester 2020 oder 
Wintersemester 2020/21 an mindestens einer Vorlesung teilgenommen haben, betrug 154 gültige Interviews.

Die angebotenen Vorlesungen sowie die dazugehörigen Übungen kombinierten in unterschiedlicher Weise synchrone und asynchrone Elemente (vgl. Tab. 1). In synchronen Formaten wurde überwiegend mit der Videokonferenzsoftware Zoom gearbeitet. Für die Bereitstellung von zuvor aufgezeichneten Videos in asynchronen Formaten sowie von (digitalen) Begleitmaterialien fand vornehmlich die universitätseigene Plattform eCampus Verwendung. In den synchron durchgeführten Vorlesungen kamen zudem immer wieder interaktive Lehrelemente zum Einsatz.

Die durchgeführte Umfrage hinsichtlich studentischer Wahrnehmungen dieses hier skizzierten Lehrangebots kam zu überaus belastbaren Ergebnissen, die auch für zukünftige Lehrplanungen wertvolle Hinweise geben: Zunächst ist zu konstatieren, dass zum einen mehrheitlich der ausdrückliche Wunsch besteht, in der Lehre zu Präsenzformaten zurückzukehren, zum anderen aber ebenso Bedarf an digitalen Lernformaten erkennbar wird, die die traditionelle Lehre weiterentwickeln. So wurde der Einsatz digitaler Vorlesungsformate grundsätzlich positiv bewertet und es wurden klare Vorteile digitaler Lehrformate herausgestellt. Zu ihnen zählen eine größere Flexibilität (sowohl räumlich als auch zeitlich, insbesondere bei asynchronen Formaten), Zeitersparnisse durch ausbleibendes Pendeln oder Raumwechseln sowie, erneut bei asynchronen Formaten, die Möglichkeit des wiederholten Abrufs entsprechender Inhalte sowie die Bestimmung eines eigenen Lerntempos. Auffallend ist zudem, dass insbesondere dann die Vorteile digitaler Vorlesungsformate betont wurden, wenn von Seiten der Dozent*innen Raum für das Stellen von Nachfragen bzw. von Diskussionsbeiträgen eingeräumt wurde. Interaktive Elemente (etwa über die Chat-Funktion, die Hand-Heben-Funktion oder auch das vorherige Einreichen von Fragen) tragen somit zur positiven Bewertung digitaler Formate bei. Insgesamt kommt in der Befragung eine deutlich kritische Haltung gegenüber traditionellen frontalen Lehrformaten zum Ausdruck, die auf einen ausgeprägten skeptischen Blick auf das klassische Vorlesungsformat im Allgemeinen schließen lässt. Hinsichtlich der Frage nach Synchronität und Asynchronität lässt sich dabei insbesondere bei Studierenden im ersten und zweiten Fachsemester eine Präferenz zu synchronen Formaten feststellen, wohingegen in späteren Studienphasen asynchrone Formate zunehmend bevorzugt werden. Eine sich im Verlauf des Studiums verbessernde Selbstorganisation sowie - für die Studierenden in Bachelorstudiengängen - die oftmals unmittelbar vorausgehenden Erfahrungen der schulischen Ausbildung können hier als Begründung vermutet werden. Dass sich diese Tendenz zu größerer Präferenz für asynchrone Formate allerdings auch für Studierende in Masterstudiengängen bestätigt, legt zudem andere Einflussfaktoren nahe, die jenseits der Fachsemesterzahl liegen und sich vielmehr auf die jeweils vermittelten Inhalte zurückführen lassen. Feststellen lässt sich indes für beide Studienabschnitte, dass eine fehlende direkte Kontaktaufnahme zu Kommiliton*innen und Dozent*innen zu mangelnder sozialer Einbindung und Vernetzung führt.

So offenbart die durchgeführte Umfrage dann auch eine ganze Reihe von als nachteilig empfundenen Elementen digitaler Vorlesungsformate. Drei Aspekte haben sich dabei besonders herauskristallisiert: Erstens wird überwiegend der fehlende Kontakt zu Kommilitonen*innen und Dozent*innen beklagt. In der Tat bleibt durch 
digitale Formate der soziale Aspekt - der Austausch mit dem Sitznachbarn oder das gemeinsame Mittagessen mit der Kommilitonin im Anschluss an die Veranstaltung - auf der Strecke, und diese für jedes Studium zentralen Elemente lassen sich auch durch fortlaufend optimierte digitale Lehrformate nicht ersetzen. Auch der Kontakt zu den jeweiligen Dozent*innen ist im digitalen Lehrbetrieb zumindest erschwert. Selbst wenn vielfältige Kontaktmöglichkeiten bestehen und auch in vielen der hier untersuchten Formate explizit eingeräumt wurden: das kurze Gespräch vor oder nach der Veranstaltung, die unverzügliche Nachfrage oder auch die direkte Rückmeldung an die Dozent*innen können ebenfalls nicht immer ohne Weiteres ins Digitale überführt werden. Zweitens attestiert die Umfrage Probleme mit und an der eigenen Lernsituation auf Seiten der Studierenden. Wurden technische Probleme überraschend selten genannt, so fanden Konzentrationsschwierigkeiten, Ablenkungen (durch erschwerte oder fehlende Trennung von Privatem und Studium) sowie Motivationsschwierigkeiten immer wieder Erwähnung. Weitere Praxis und Übung vermögen hier in Zukunft zwar möglicherweise zur Verbesserung und wachsender Erfahrung und Routine beizutragen, doch auch hier zeigt sich deutlich, dass sich die (Lern-)Atmosphäre des Hörsaals oder des Seminarraums nicht digital abbilden lässt. Unaufgelöst bleiben zum Teil auftretende Widersprüche zwischen den hier skizzierten Problemen auf der einen und den wahrgenommenen Freiheiten und Annehmlichkeiten der Studienorganisation auf der anderen Seite. Drittens wurde wiederholt ein als zu gering empfundenes Maß an studentischer Beteiligungs- und Diskussionsmöglichkeit zum Ausdruck gebracht. Zwar kann der Veranstaltungstypus Vorlesung als traditionell frontales Format gelten, in dem, insbesondere in Einführungsveranstaltungen in niedrigen Fachsemestern, die Vermittlung von Wissen im Vordergrund steht, doch tragen digitale Formate gewiss dazu bei, dass verstärkt über Weiterentwicklungen diskutiert werden kann. Da diese Befunde an eine Debatte anschließen, die bereits vor der durch die Pandemie bedingten ,Zwangsdigitalisierung“ des Lehrbetriebs geführt wurde, kommt der Corona-Krise eine Katalysatorrolle hinsichtlich der Weiterentwicklung des Vorlesungsformates zu.

Insgesamt bleibt bezüglich der Ergebnisse der Umfrage festzuhalten, dass von den befragten Bonner Studierenden im offenen Frageblock mehr Studierende Vorteile $(n=124)$ als Nachteile $(n=102)$ digitaler Formate angaben. Hinsichtlich der explizit erfragten Wünsche für die post-pandemische Lehre ist zu konstatieren, dass die Studierenden auch für die Zukunft eine Kombination synchroner und asynchroner Lehrformate bevorzugen. Ergänzt um die als zentral identifizierten Interaktions- und Partizipationsmöglichkeiten sowie die Bereitstellung von Lernaufgaben und -materialien zur Vor- und Nachbereitung über entsprechende Lernplattformen, kann durch hybride Formate in der Tat das Beste aus beiden Welten - der digitalen und der Präsenzlehre - in den künftigen Lehralltag, zumal im Veranstaltungsformat Vorlesung, integriert und kombiniert werden. Zudem können digital verfügbare wissensvermittelnde Vorlesungsinhalte dazu beitragen, weniger leseaffine Lerntypen gerade zu Beginn des Studiums zu unterstützen.

Anhand der durchgeführten Umfrage konnte dabei, trotz aller unterstrichener Vorteile digitaler Formate und des Wunsches nach Hybridlösungen, eine ,,analoge Sehnsucht" attestiert werden. In der Tat gilt es, den nun über mehrere Semester praktizierten digitalen Lehrbetrieb in Zukunft wieder behutsam in Präsenzformate 
zu überführen, und so die zentrale Aufgabe der Hochschulen zu wahren, die eben nicht nur im „Informations-Download aufs Hirn“ (Fbn. 136) besteht, sondern weit darüber hinausgeht. Das Studium, noch dazu im Bereich Geisteswissenschaft, findet eben nicht nur im Vorlesungssaal oder Seminarraum statt, sondern erstreckt sich auch über das vielfältige, gemeinsame Universitätsleben, den Besuch der Mensa, außercurriculare Aktivitäten und Angebote auf dem Campus und anderorts.

Und dennoch: Vorlesungsformate, mit ihrer klassischen frontalen Ausrichtung, können auch in Zukunft besonders von digitalen Elementen profitieren, wobei begleitende und vertiefende Übungen (sowie Seminare) weiterhin besonderen Nutzen aus den unzweifelhaften Vorteilen der Präsenzlehre ziehen können. Zusätzlich zu dieser Einschätzung lässt sich aus den Umfrageergebnissen ableiten: Das letztlich zentrale Kriterium ,guter“ Hochschullehre ist weniger im Format (digital, Präsenz oder hybrid) zu suchen, als vielmehr in Qualität und Zusammenspiel von Inhalt und Umsetzung. Damit knüpft die hier vorgelegte Untersuchung an Ergebnisse einer bereits vor der Pandemie einsetzenden Debatte an: Inhalt und Form der Lehrformate müssen stimmig sein, sich gewinnbringend ergänzen und unterstützen. Folglich sollte die Digitalisierung des Lehrbetriebs nicht als neues „Normal“" wahrgenommen, sondern vielmehr als ein Praxistest verstanden werden, aus dem auch für die Zukunft Erfahrungswerte gewonnen werden können.

Funding Open Access funding enabled and organized by Projekt DEAL.

Open Access Dieser Artikel wird unter der Creative Commons Namensnennung 4.0 International Lizenz veröffentlicht, welche die Nutzung, Vervielfältigung, Bearbeitung, Verbreitung und Wiedergabe in jeglichem Medium und Format erlaubt, sofern Sie den/die ursprünglichen Autor(en) und die Quelle ordnungsgemäß nennen, einen Link zur Creative Commons Lizenz beifügen und angeben, ob Änderungen vorgenommen wurden.

Die in diesem Artikel enthaltenen Bilder und sonstiges Drittmaterial unterliegen ebenfalls der genannten Creative Commons Lizenz, sofern sich aus der Abbildungslegende nichts anderes ergibt. Sofern das betreffende Material nicht unter der genannten Creative Commons Lizenz steht und die betreffende Handlung nicht nach gesetzlichen Vorschriften erlaubt ist, ist für die oben aufgeführten Weiterverwendungen des Materials die Einwilligung des jeweiligen Rechteinhabers einzuholen.

Weitere Details zur Lizenz entnehmen Sie bitte der Lizenzinformation auf http://creativecommons.org/ licenses/by/4.0/deed.de.

\section{Literatur}

Becker, Manuel, et al. 2020. Rückblick auf das erste „Corona-Semester“. Ergebnisse einer semesterbegleitenden Untersuchung der Task Force Digitale Lehre des Instituts für Politische Wissenschaft und Soziologie der Universität Bonn. https://link.springer.com/article/10.1007/s41358-020-00243-

2. Zeitschrift für Politikwissenschaft 4 (2020). Zugegriffen: 16.08.2021.

Looß, Maike. 2019. Die erstaunliche Unhaltbarkeit einer unhaltbaren Theorie. Education Permanente $1: 15-18$.

Wisniewski, Benedikt. 2013. Der Unsinn von den Sinnen. In Schule auf Abwegen. Mythen, Irrtümer und Aberglaube in der Pädagogik, Hrsg. Benedikt Wisniewski, Andreas Vogel, 11-26. Baltmannsweiler: Schneider Hohengehren. 